\title{
Endogenous regulation of a therapeutic transgene restores homeostasis in arthritic joints
}

\author{
A.V. Miagkov, ${ }^{1}$ A.W. Varley, ${ }^{2}$ R.S. Munford ${ }^{2}$ and S.S. Makarov ${ }^{1,3,4}$ \\ ${ }^{1}$ Thurston Arthritis Research Center, University of North Carolina at Chapel Hill, Chapel Hill, North Carolina, USA \\ ${ }^{2}$ Molecular Host Defense Lab, Division of Infectious Diseases, Department of Internal Medicine, \\ University of Texas Southwestern Medical Center, Dallas, Texas, USA \\ ${ }^{3}$ Center for Inflammatory Disorders, and \\ ${ }^{4}$ Department of Endodontics, University of North Carolina at Chapel Hill, Chapel Hill, North Carolina, USA
}

Address correspondence to: Sergei Makarov, 4109 Thurston, Campus Box 7280,

University of North Carolina at Chapel Hill, Chapel Hill, North Carolina 27599, USA.

Phone: (919) 966-9565; Fax: (919) 966-1739; E-mail: smak@med.unc.edu.

A.V. Miagkov's present address is: Department of Neurology, Johns Hopkins School of Medicine, Baltimore, Maryland, USA.

Received for publication November 1, 2001, and accepted in revised form March 18, 2002.

\begin{abstract}
The treatment of chronic inflammatory diseases is complicated by their unpredictable, relapsing clinical course. Here, we describe a new strategy in which an inflammation-regulated therapeutic transgene is introduced into the joints to prevent recurrence of arthritis. To this end, we designed a recombinant adenoviral vector containing a two-component, inflammation-inducible promoter controlling the expression of human IL-10 (hIL-10) cDNA. When tested in vitro, this system had a low-level basal activity and was activated four to five orders of magnitude by various inflammatory stimuli, including TNF- $\alpha$, IL-1 $\beta$, IL- 6 , and LPS. When introduced in joints of rats with recurrent streptococcal cell wall-induced arthritis, the IL-10 transgene was induced in parallel with disease recurrence and effectively prevented the influx of inflammatory cells and the associated swelling of the joints. Levels of inflammation-inducible hIL-10 protein within the joints correlated closely with the severity of recurrence. An endogenously regulated therapeutic transgene can thus establish negative feedback and restore homeostasis in vivo while minimizing host exposure to the recombinant drug.
\end{abstract}

J. Clin. Invest. 109:1223-1229 (2002). DOI:10.1172/JCI200214536.

\section{Introduction}

Rheumatoid arthritis is a prototypical chronic inflammatory disease characterized by an imbalance of proand anti-inflammatory mediators. Gene therapy has been proposed as a means to restore impaired balance by introducing anti-inflammatory genes into arthritic joints (1). Studies in animal models have shown that gene transfer technology is uniquely suited for therapeutic protein delivery in rheumatoid arthritis (2); two phase I clinical trials in humans are currently underway (3). A major challenge for the anti-inflammatory gene therapy approach remains, however. Since rheumatoid arthritis typically progresses through cycles of relapse and remission, the production of a therapeutic protein must be regulated in order to match the recurrent course of the disease.

A number of vectors with drug-inducible promoters for achieving regulatable transgene expression have been described (4-8). In this approach, transgene expression in vivo is regulated by an orally bioavailable small-molecule drug. However, due to the unpredictable course of the disease, this kind of system will require long-term, largely empirical drug adjustments made by the physician or the patient. Overproduction of anti-inflammatory molecules may be associated with tachyphylaxis, tissue toxicity, or enhanced susceptibility to infection. In addition, experimental evidence indicates that inappropriate timing of anti-inflammatory protein administration can undermine the efficacy of treatment (9). To avoid these problems, we proposed an alternative approach that we call physiologically responsive gene therapy (PRGT) (10). In this approach, anti-inflammatory transgene expression is autonomously regulated by an inflammation-inducible promoter. In this study we assessed the feasibility of using PRGT for treatment of recurrent arthritis. In preliminary studies, we developed a two-component expression system that responds vigorously to inflammatory stimuli in vivo (11). Here, we constructed an adenoviral vector carrying the cDNA of a pleiotropic anti-inflammatory cytokine, human IL-10 (hIL-10), driven by the inflammation-inducible promoter. We demonstrate that intra-articular (IA) delivery of inflammationregulated hIL-10 effectively prevents recurrence of streptococcal cell wall (SCW) arthritis in rats. The recombinant therapeutic protein is produced in direct relationship to the onset, severity, and duration of inflammation, thus indicating homeostatic regulation of the therapeutic transgene.

\section{Methods}

SCW arthritis model. Female Lewis rats were obtained from Charles River Laboratories (Wilmington, Massa- 
chusetts, USA) and used in the SCW reactivation model of arthritis. In this model, an acute, transient, monoarticular arthritis is produced by IA injection of SCW fragments isolated from group A streptococci. The primary structural components of the SCW fragments are polymers of peptidoglycan-polysaccharide (PG-APS). Several weeks after the IA injection, an intravenous injection of PG-APS induces reactivation of chronic inflammatory arthritis in previously injured joints (12). To assess joint inflammation, the lateral diameters of ankle joints were measured in triplicate using a digital caliper, as previously described $(9,13)$. To reduce bias, all animals were coded and measurements were done randomly.

For synovial lavages, ankles were excised and two needles were inserted into the joint cavity. A 30-gauge needle was used for the inflow of perfusion solution ( $0.5 \mathrm{ml}$ of PBS), and a 22-gauge needle was used for collecting the perfusate. After centrifugation, supernatants were frozen for subsequent quantification of hIL-10. Sedimented cells were resuspended, red blood cells were lysed, and white blood cells were counted using a Coulter counter (Beckman Coulter Inc., Miami, Florida, USA).

To measure in vivo luciferase expression, excised ankle joints were pulverized in liquid nitrogen and extracted for 30 minutes on ice with $1 \mathrm{ml}$ of an extraction buffer $(0.25 \mathrm{M}$ Tris at $\mathrm{pH} 7.5,10 \mathrm{mM}$ EDTA, $1 \mathrm{mM}$ EGTA, $1 \mathrm{mM}$ PMSF, $2.5 \mu \mathrm{g} / \mathrm{ml}$ aprotinin, $5 \mu \mathrm{g} / \mathrm{ml}$ leupeptin, and $2.5 \mu \mathrm{g} / \mathrm{ml}$ pepstatin). Luciferase activity in the extracts was determined using a luminometer. The possible influence of quenchers or enhancers of luciferase activity in the samples was controlled by determining chemiluminescence of recombinant luciferase in the presence of joint extracts.

Adenovirus preparation and storage. Adenoviruses (Ad) were produced and purified as described (11). After titration, each virus was frozen and stored at $-70^{\circ} \mathrm{C}$. Immediately prior to IA injections, the viruses were thawed and diluted in saline. In two in vivo experiments and most of the in vitro studies, Ad viruses were stored in $67 \mathrm{mM} \mathrm{NaCl}, 2.5 \mathrm{mM} \mathrm{KCl}, 0.5 \mathrm{mM}$ $\mathrm{MgCl}_{2}, 10 \mathrm{mM}$ Tris ( $\mathrm{pH} 7.4$ ), and $50 \%$ glycerol at $-20^{\circ} \mathrm{C}$ for up to 3 months. Unless otherwise noted, Ad were injected intra-articularly at day -2 , at $10^{7}$ plaqueforming units (PFU) per injection. When compared with sham-injected joints, injection of reporter vectors (Ad.C3-tat/HIV-luc and Ad.RR5) caused an increase in joint diameter from day -2 to day 0 but did not influence the severity of recurrence induced by PG-APS injection at day 0.

Cell culture. Primary rat synovial fibroblasts were obtained by enzymatic dispersal of synovial explants from rats with SCW arthritis (13). Cells were maintained in RPMI 1640 with $15 \%$ FBS and used at passages 5-10.

Immunodetection methods. Cell lysates were resolved on a denaturing $15 \%$ SDS-polyacrylamide gel and transferred to nitrocellulose. hIL-10 was detected with a polyclonal primary hIL-10 antibody sc-1273 $(1: 1,000)$ (Santa Cruz Biotechnology Inc., Santa Cruz, California, USA). Immune complexes were visualized using secondary horseradish peroxidase-conjugated antibody $(1: 3,000)$ and a Renaissance ECL kit (NEN Life Science Products Inc., Boston, Massachusetts, USA). To assess hIL-10 in vivo, synovial lavages were pooled (four joints at each time point), forty microliters of the mixture were resolved on SDS-polyacrylamide gel and immunodetected with hIL-10 antibody, as above. Human recombinant IL-10 (Biosource International, Camarillo, California, USA) provided a positive control. hIL-10 ELISA was performed on coded samples using antibodies and recombinant hIL-10 from Pharmingen (San Diego, California, USA).

\section{Results}

The two-component C3-tat/HIV promoter is a broadly responsive sensor of chronic inflammation. In order to achieve physiological regulation of anti-inflammatory transgenes, a promoter element should satisfy certain criteria. First, it should respond to the principal proinflammatory mediators. Second, to provide a large dynamic range of responses, it should have low-level basal activity and high inducibility. Finally, the fully activated promoter should be able to drive the production of sufficient amounts of recombinant protein to achieve a therapeutic effect. Transcription of many acute-phase protein (APP) genes is controlled by inflammation-inducible promoters, which respond to inflammatory stimuli in a dose-dependent fashion. However, the absolute levels of expression achievable by vectors with endogenous APP gene promoters are disappointingly low compared with levels provided by the strong constitutive cytomegalovirus (CMV) promoter (14). In our previous studies, we developed a two-component inflammation-inducible expression system. This chimeric construct uses the promoter of the complement factor 3 (C3) APP gene to control the expression of the HIV Tat protein; in the same construct, Tat transactivates the expression of a transgene $(\mathrm{rX})$ linked to the HIV promoter (11) (Figure 1). Since both the C3 and HIV promoters are cytokine responsive, this binary construct allows both cis and trans activation of $\mathrm{rX}$ production by inflammatory mediators. When this binary element was incorporated into a nonreplicating Ad and injected into mice, it exhibited high inducibility in several tissues in response to bacterial endotoxin and turpentine oil, and after induction it attained levels of $\mathrm{rX}$ expression comparable to those provided by the CMV promoter (11).

Here we first examined the ability of the C3-tat/HIV promoter to respond in vitro to important mediators of chronic inflammation. Primary synovial fibroblasts derived from arthritic rat joints were infected with a reporter Ad (Ad.C3-tat/HIV-luc). Control cells were infected with the empty vector (Ad.RR5) or with an Ad that produces luciferase under the control of the CMV promoter (Ad.CMV-luc). Following adenoviral infec- 


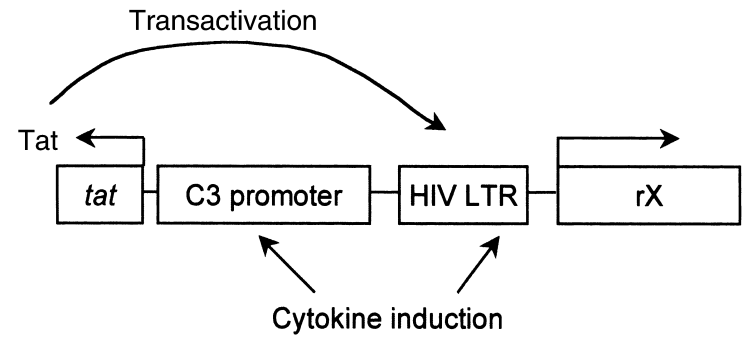

Figure 1

The two-component C3-tat/HIV promoter. LTR, long terminal repeat.

tion, serum-starved cells were stimulated with LPS (10 $\mu \mathrm{g} / \mathrm{ml}$ ), IL-1, IL-6, or TNF- $\alpha$ (each cytokine was used at a concentration of $20 \mathrm{ng} / \mathrm{ml}$ ). The stimuli activated C3-tat/HIV promoter by approximately four to five orders of magnitude, and the peak levels were comparable to those provided by the CMV promoter (Figure 2 , $a$ and $b$ ). Of note, CMV-driven luciferase expression was also activated by these stimuli, although the range of induction (approximately twofold) was far less than was seen with the C3-tat/HIV promoter. As IL-1, IL-6, and TNF- $\alpha$ are considered the pivotal cytokines in chronic inflammation, the C3-tat/HIV element thus appears to be a broadly responsive "sensor" of inflammation with a very large dynamic range.

Recurrence of SCW arthritis induces activation of C3-tat/ HIV promoter in vivo. To assess the inducibility of the C3-tat/HIV promoter by chronic inflammation in joints in vivo, we first examined the expression of the luciferase reporter during the reactivation phase of SCW arthritis in rats. A distinct feature of this model is the predictability of the recurrence, which reaches a

\section{Figure 2}

The two-component C3-tat/HIV promoter is a broadly responsive sensor of chronic inflammation. ( $\mathbf{a}$ and $\mathbf{b}$ ) Induction by the principal proinflammatory cytokines in vitro. Primary synovial fibroblasts were infected in vitro by incubating overnight with the indicated Ad vectors at a multiplicity of infection of 100 . Twenty-four hours later, cells were starved in $0.5 \%$ FBS medium for 24-48 hours, followed by stimulation for 6 hours with the indicated cytokines $(20 \mathrm{ng} / \mathrm{ml})$ or LPS $(10 \mu \mathrm{g} / \mathrm{ml})$. Luciferase activity in cell lysates was normalized to total protein. (a) The levels of inflammation-inducible C3-tat/HIV-driven expression are comparable to those attainable with the CMV promoter. Data are presented as mean of duplicate measurements within an individual experiment; error bars show SD. (b) The C3-tat/HIV promoter provides a large dynamic range of response. The values of fold induction were calculated as the average of readings in independent experiments performed in duplicate (two experiments with IL- 1 and IL- 6 and three experiments with TNF- $\alpha$ and LPS). (c) In vivo induction following reactivation of SCW arthritis. On day -42 , animals were primed by IA injection of PG-APS in both ankle joints. The same joints were injected with the indicated Ad vectors (107 PFU in $10 \mu \mathrm{l}$ ) on day -2 . On day 0 , arthritis was reactivated by an intravenous injection of PG-APS. Luciferase activity was measured in extracts of pulverized joints (each bar shows the mean \pm SD of two joints). In three independent experiments, luciferase activity peaked on day 2-4 and remained elevated through day 4. Unstim., unstimulated; IV, intravenous; rlu, relative luciferase units. peak at 2 to 4 days and gradually subsides thereafter (12). Ad.C3-tat/HIV-luc and control Ad vectors were injected into primed ankle joints 2 days prior to reactivation of SCW arthritis. In the absence of reactivation, the luciferase activity in the joints injected with Ad.C3tat/HIV-luc was low, but following reactivation, it achieved the levels attainable with the constitutive CMV promoter (Figure 2c). In three independent experiments, luciferase activity peaked at 2 to 4 days after reactivation; the maximal induction was in the range of 18- to 80-fold compared with unreactivated joints.

Inflammation-inducible production of hIL-10 in vitro. When considering the candidacy of a transgene for a therapeutic protocol, we reasoned that the transgene product should inhibit the numerous inflammatory pathways acting upon the inducible promoter; otherwise, the inducible promoter might remain activated by unchecked proinflammatory mediators. In previous studies we determined that simultaneous suppression of many inflammatory responses, including the induction of IL-1, IL- 6 , and TNF- $\alpha$, can be achieved by blocking activation of the transcription factor NF- $\kappa B$ (15, 16). Anti-inflammatory cytokine IL-10 is a potent extracellular antagonist of NF- $\kappa B$ activation $(17,18)$; in addition, several recent reports have demonstrated the therapeutic efficacy of unregulated IL-10 gene transfer in animal models of rheumatoid arthritis
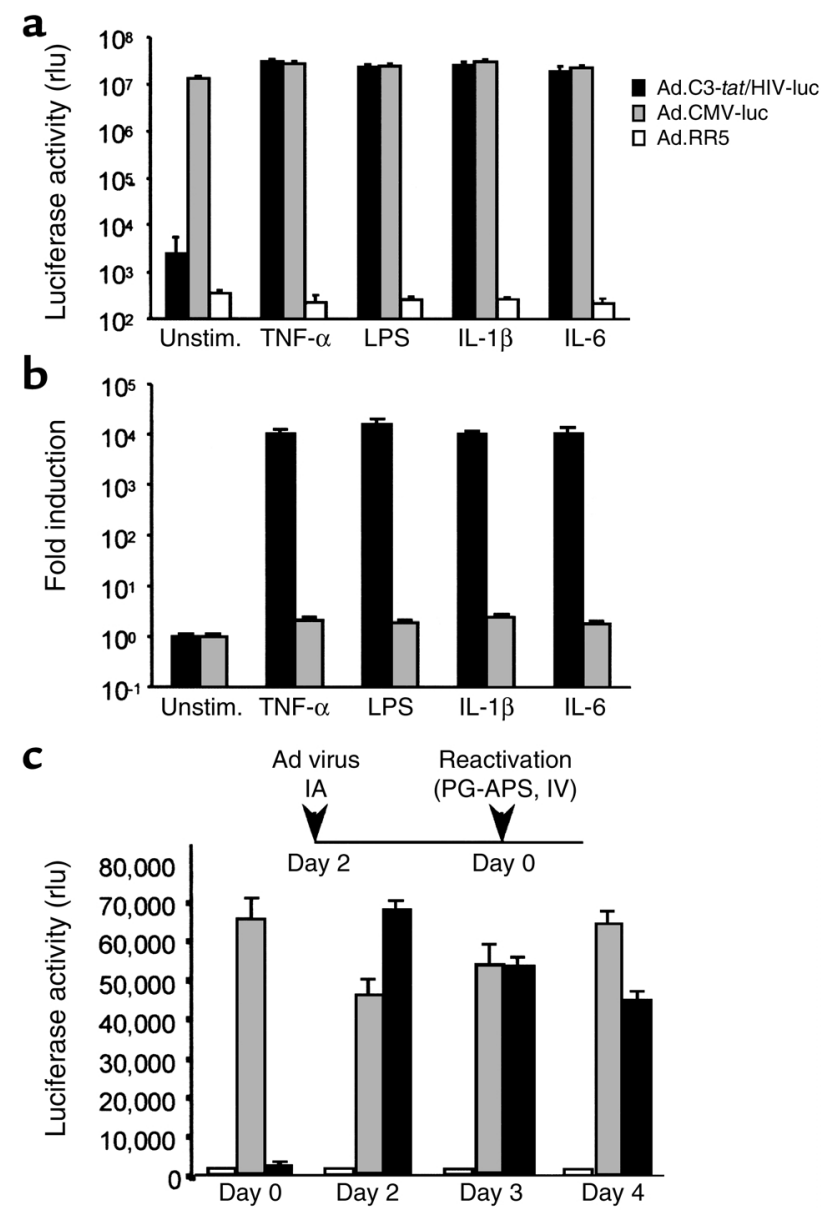

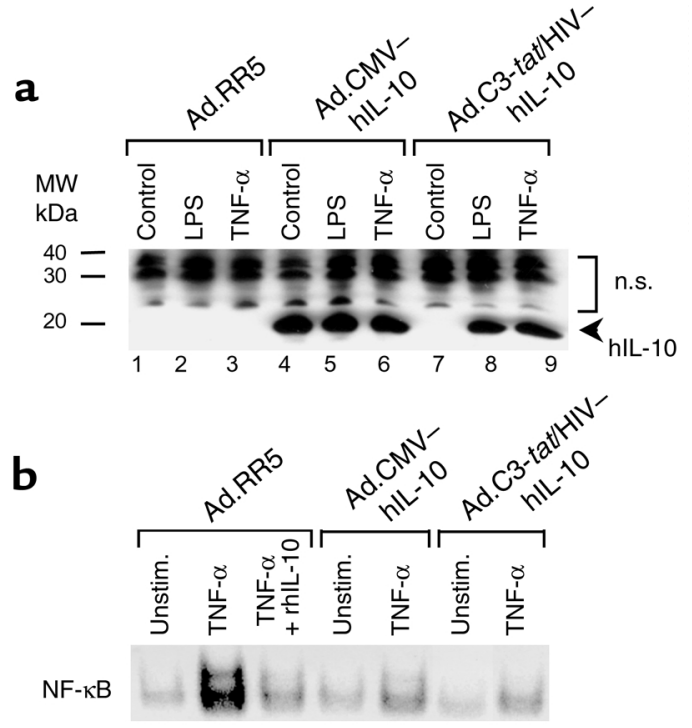

\section{Figure 3}

(a) Inflammation-inducible production of hIL-10 in primary synovial fibroblasts in vitro. Cells were infected as for Figure $2 \mathrm{a}$ and stimulated with LPS $(1 \mu \mathrm{g} / \mathrm{ml})$ or TNF- $\alpha(10 \mathrm{ng} / \mathrm{ml})$ for 48 hours. The levels of hIL-10 in conditioned media were assessed by Western blotting. The hIL-10 band was identified by comparison with a recombinant hIL-10 standard (not shown). Data representative of two independent experiments are shown. (b) Adenoviral hIL-10 inhibits NF-KB activation in vitro. Conditioned medium from TNF- $\alpha$-stimulated infected cells (shown in a, lanes 3, 6, and 9) was added to uninfected primary synovial fibroblasts; where indicated, recombinant hIL-10 (rhIL-10; $100 \mathrm{ng} / \mathrm{ml}$ ) was also added. After 10 minutes of preincubation, TNF- $\alpha(100 \mathrm{ng} / \mathrm{ml})$ was added for a 15 -minute incubation period. NF-KB DNA-binding activity in nuclear extracts was analyzed by electromobility shift assay (EMSA). NS, nonspecific bands.

(19-23). We therefore constructed an adenoviral vector (Ad.C3-tat/HIV-hIL-10) expressing the cDNA of hIL-10. Control viruses were Ad.C3-tat/HIV-luc, the empty vector Ad.RR5, and Ad.CMV-hIL-10, an Ad that produces hIL-10 constitutively under the control of the CMV promoter. Synovial cells infected in vitro with Ad.C3-tat/HIV-hIL-10 produced undetectable amounts of hIL-10, whereas treatment with TNF- $\alpha$ or LPS resulted in secretion of hIL-10 in concentrations similar to those produced by cells infected with Ad.CMV-hIL-10 (Figure 3a). The secreted hIL-10 was biologically active: it effectively antagonized induction of NF- $\kappa B$ DNA-binding activity in primary synoviocytes in response to TNF- $\alpha$ (Figure $3 \mathrm{~b}$ ), prevented activation of NF- $\kappa B$ reporter gene expression in TNF- $\alpha-$ and LPS-stimulated L929 fibroblasts (not shown), and suppressed IL- 6 production by LPS-stimulated murine macrophages (not shown).

Gene transfer of inflammation-inducible bIL-10 prevents reactivation of $S C W$ arthritis. To assess the therapeutic efficacy of inflammation-inducible hIL-10 expression, PG-APS-primed rat ankle joints were injected with Ad.C3-tat/HIV-hIL-10 or control vectors 1-2 days before arthritis was reactivated by an intravenous injection of PG-APS. The severity of inflammation after reactivation was assessed by measuring joint swelling $(13,16)$ and the numbers of leukocytes within the joints (24). As determined in two independent experiments, gene transfer of inflammation-inducible hIL-10 afforded efficacious protection against reactivationinduced leukocytosis (Figure 4a and data not shown). Inflammation-inducible hIL-10 gene transfer also significantly inhibited joint swelling (Figure 4b). Inhibition of joint swelling by Ad.C3-tat/HIV-hIL-10 gene transfer was statistically significant in four independent experiments (Table 1). In one experiment, Ad.C3tat/HIV-hIL-10 gene transfer did not result in statistically significant suppression of joint swelling $(P>0.05)$.

Homeostatic regulation of inflammation-inducible bIL-10 expression in vivo. Quantitative assessments of hIL-10 in joint lavages by ELISA showed that, in the absence of reactivation, hIL-10 levels in joints injected with Ad.C3tat/HIV-hIL-10 were at the lower limit of detection (1 $\mathrm{ng} / \mathrm{ml}$ ). Reactivation of arthritis caused striking increases in hIL-10 levels on days 1 and 2, returning to nearbaseline (unreactivated) levels on day 4 (Figure 5a). Since ELISA readings can be influenced by various inhibitors often found in biological fluids, we additionally examined hIL-10 expression using Western blotting. As shown in Figure 5b, qualitative assessments based on Western blotting were in good agreement with the ELISA readings. In contrast to the joints injected with Ad.C3-tat/HIV-hIL-10, hIL-10 production from Ad.CMV-hIL-10 persisted at a constant level throughout the observation period (Figure $5 \mathrm{~b}$ ). The observed sustained expression of Ad.CMV-hIL-10 is consistent with our previous observation that the expression of a CMV-driven reporter green fluorescence protein persisted for at least 1 week after Ad gene transfer into arthritic rat joints (16). This refutes the possibility that the shutoff of inflammation-inducible hIL-10 expression at day 4 was a consequence of an immune response directed against Ad-transduced cells. Another possible explanation is that suppression of the antiapoptotic NF- $\kappa B$ pathway (16) by IL-10 might lead to elimination of Ad-infected cells; however, persistence of CMV-driven hIL-10 expression (Figure 5b) argues against this possibility. Furthermore, inflammation-inducible luciferase expression persisted through day 4 (Figure 2c), strongly suggesting that the rapid decline of Ad.C3tat/HIV-hIL-10 expression was a direct consequence of hIL-10-mediated inhibition of inflammation, with subsequent downregulation of the inflammation-inducible promoter. We therefore conclude that gene transfer mediated by Ad.C3-tat/HIV-hIL-10 establishes a negative feedback loop similar to those involved in homeostatic regulation of endogenous inflammatory genes.

The severity of recurrent SCW arthritis in rats can be regulated by varying the doses of intra-articularly and intravenously injected PG-APS and the interval between priming and reactivation (12). This allowed us to investigate the relationship between the severity of recurrent arthritis and IA production of inflammation-inducible hIL-10. In three independent experi- 

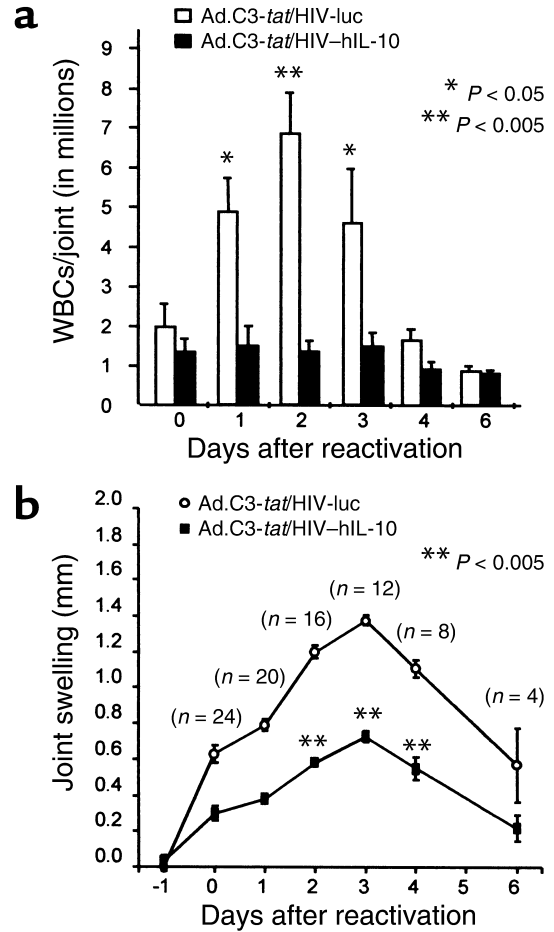

ments, IA hIL-10 levels in joints injected with Ad.C3tat/HIV-hIL-10 increased 16-, 70-, and 288-fold above baseline by day 2-4 (Table 1 ). The maximal fold induction in hIL-10 concentration was directly related to the severity of SCW arthritis in the corresponding control groups $(r=0.93)$ (Figure $5 c$ ). In those experiments, IA hIL-10 levels returned to near baseline by day 4 . These data suggest strongly that inflammation-inducible expression of hIL-10 occurred in direct relationship to the intensity and duration of inflammation.

\section{Discussion}

The objective of our work was to test the concept of PRGT for treating a recurrence of chronic inflammation. The benefits of the approach seem self-evident, but the feasibility of self-regulated anti-inflammatory gene therapy is yet to be established. Numerous transgenes have been shown to prevent experimental arthri-

\section{Figure 4}

Gene transfer of inflammation-inducible hIL-10 prevents reactivation of SCW arthritis. (a) Inhibition of leukocytosis. On day -42 , animals were IA injected with PG-APS in both ankle joints. On day -1 , animals were injected in both ankle joints with the indicated Ad, as for Figure $2 b$. In this experiment there were 12 animals per group on day -1 . On day 0 , reactivation was induced by intravenous injection of PG-APS. On days $0,1,2,3,4$, and 6 after reactivation, two animals from each group were euthanized, and ankle joints were perfused for determining the numbers of infiltrating white blood cells (WBCs) (21). The time course of leukocytosis is shown. Each bar shows the mean \pm SEM of measurements from four joints. Data representative of two experiments are shown. (b) Inhibition of joint swelling. In parallel to measuring leukocytosis, inflammation was assessed by measuring joint swelling. As animals in each group were euthanized in order to obtain joint lavages, the data points represent a diminishing number of joints. Swelling was calculated as $\left(d_{i}\right)-\left(d_{0}\right)$, where $\left(d_{i}\right)$ and $\left(d_{0}\right)$ are mean joint diameters at day $i$ and day 0 . Error bars represent SEM. $P$ values for comparisons between groups in $\mathbf{a}$ and $\mathbf{b}$ were calculated using the unpaired one-tailed Student $t$ test. Data on inhibition of joint swelling in other experiments are presented in Table 1. tis, including IL-10 (19-23), soluble TNF receptors, IL-1 receptor antagonist, Fas ligand, and TGF- $\beta$ (13, 25-29), and several inflammation-inducible promoters have been described $(10,14,30,31)$. However, it was not at all clear whether an autoregulated system would work to prevent inflammation in vivo. The fact that our two-component inflammation-inducible construct responded to a high concentration of systemic inflammatory stimuli (e.g., LPS and turpentine) was encouraging, but this did not guarantee that the promoter would similarly respond to the recurrence of chronic inflammation, or that this would work in the context of the synovium. It was also not at all clear whether the promoter could induce the expression of tangible amounts of therapeutic proteins. We also did not know if the inducible IL-10 production would be beneficial, because inappropriate timing can diminish the efficacy of anti-inflammatory proteins (9).

Table 1

Therapeutic efficacy of inflammation-inducible hIL-10 gene transfer in SCW arthritis

\begin{tabular}{|c|c|c|c|c|}
\hline Experiment & Ad vector & AMax. swelling $(\mathrm{mm}) \pm \mathrm{SEM}$ & ${ }^{B}$ Inhibition of swelling $(\%)^{\mathrm{C}}(P$ value $)$ & DMax. hIL-10 induction (fold) \\
\hline 1 & $\begin{array}{c}\text { None } \\
\text { Ad.C3-tat/HIV-luc } \\
\text { Ad.C3-tat/HIV-hIL-10 }\end{array}$ & $\begin{array}{l}1.13 \pm 0.25(n=10) \\
1.28 \pm 0.16(n=10) \\
0.53 \pm 0.21(n=12)\end{array}$ & E59\% $(P<0.01)$ & ND \\
\hline 2 & $\begin{array}{c}\text { Ad.C3-tat/HIV-luc } \\
\text { Ad.C3-tat/HIV-hIL-10 }\end{array}$ & $\begin{array}{l}1.61 \pm 0.17(n=4) \\
0.76 \pm 0.29(n=4)\end{array}$ & $53 \%(P<0.05)$ & 288 \\
\hline 3 & $\begin{array}{c}\text { Ad.RR5 } \\
\text { Ad.C3-tat/HIV-hIL-10 }\end{array}$ & $\begin{array}{r}0.91 \pm 0.15(n=12) \\
-0.25 \pm 0.07(n=12)\end{array}$ & $100 \%(P<0.005)$ & 16 \\
\hline 4 & $\begin{array}{c}\text { Ad.C3-tat/HIV-luc } \\
\text { Ad.C3-tat/HIV-hIL-10 }\end{array}$ & $\begin{array}{l}0.75 \pm 0.05(n=12) \\
0.43 \pm 0.04(n=12)\end{array}$ & $43 \%(P<0.005)$ & 70 \\
\hline
\end{tabular}

${ }^{\mathrm{A} C a l c u l a t e d}$ as joint swelling in relation to day $0: \Delta=\left(d_{\max }\right)-\left(d_{0}\right)$, where $\left(d_{\max }\right)$ is the maximal joint diameter throughout the observation period, and $\left(d_{0}\right)$ is joint diameter at day 0 (average of $n$ joints). ${ }^{B}$ Calculated as $\left.\left(\Delta_{\text {control group }}-\Delta_{\text {experimental group }}\right) / \Delta_{\text {control group }}\right) \times 100 \%$. ${ }^{C}$ The significance of difference in reactivation-induced joint swelling between groups was calculated using the unpaired one-tailed Student $t$ test. DMean values of IA hIL-10 were calculated as the average of four joints per group. Maximal values of hIL-10 induction throughout the observation period are shown. ECompared with Ad.C3-tat/HIV-luc group. Max., maximal. ND, not determined. 
a

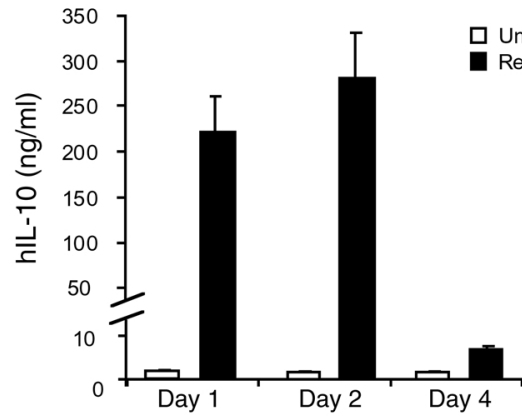

b

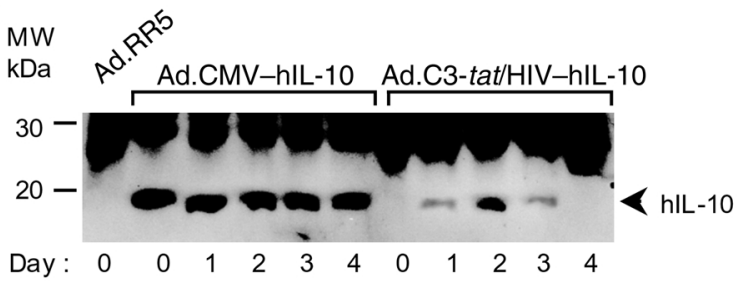

C

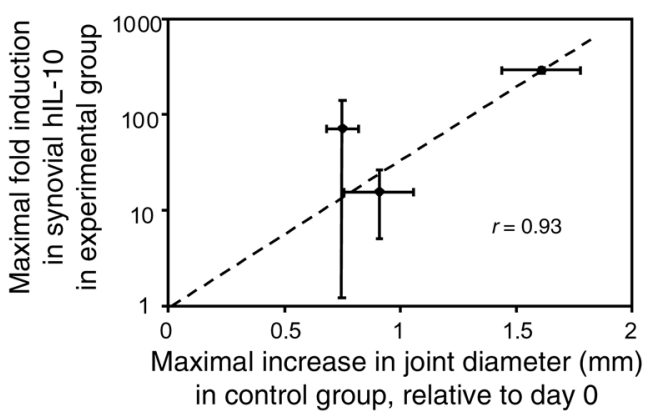

In this context, the novelty and the significance of our work is the demonstration that autoregulatory gene therapy for rheumatoid arthritis is feasible. A distinct advantage of this strategy is that it inhibits inflammation while carrying minimal risk of tissue toxicity or tachyphylaxis from persistent overproduction of the transgene product(s). In this method, compared with other methods for anti-inflammatory drug administration, the risk of unwanted immunosuppression can also be reduced: the drug may be made when, where, in the necessary amount, and for only as long as is necessary for the control of local inflammation.

Our study represents proof of principle rather than a description of a construct immediately available for clinical use. The most serious practical issue remains the selection of a vector for inflammation-inducible gene delivery. Ad vectors are known to induce inflammation within the joint approximately 2 weeks after Ad infection. This limitation precluded examination of the long-term effects of inflammation-inducible expression in the present study. In this regard, use of low-immunogenic vectors such as adeno-associated virus-based vectors (32) may be advantageous, especially because adeno-associated virus-based vector expression is reportedly elevated in the inflamed joints (33-35).

Another concern was that the expression of Tat in our promoter might cause unwanted effects, e.g., cytotoxicity, immunogenicity, and inflammation. We investigat-

\section{Figure 5}

The expression of inducible hIL-10 transgene in vivo. (a) Reactivated vs. unreactivated joints. Animals with PG-APS-preinjured ankle joints received IA Ad.C3-tat/HIV-hIL-10 on day-2. Arthritis was reactivated in half the animals by intravenous PG-APS (black bars) on day 0 . The other half was sham-injected with saline (white bars). The concentrations of hIL-10 in joint perfusates were assessed by ELISA. Each bar shows the mean \pm SEM of four joints. In the unreactivated group, $\mathrm{hIL}-10$ concentrations were at or below the limit of detection $(1 \mathrm{ng} / \mathrm{ml})$. In a control group (Ad.C3-tat/HIV-luc injected animals), the expression of luciferase has reached a peak at day 4 after reactivation (data not shown). (b) Constitutive vs. inducible transgene expression. In a similar experiment, animals were IA injected at day -2 with the constitutive Ad.CMV-hIL-10 or inducible Ad.C3-tat/HIV-hIL-10. Following reactivation of arthritis (day 0 ), hIL-10 levels in joint lavages were assessed by Western blotting. Ad.RR5: perfusates of joints injected with an empty Ad. Each lane represents a pool of four joints. In a parallel control experiment, animals were IA injected with Ad.C3-tat/HIV-luc reporter, which expression persisted through day 4 (data represented by Figure 2c). (c) Levels of inflammation-induced hIL-10 correlate with the severity of arthritis. Each point represents data from an independent experiment and is the average of four or more joints (see Table 1); error bars equal \pm SEM. Abscissa: maximal SCW-induced swelling in the counterpart control animals (injected with empty or with reporter Ad). Ordinate: maximal average hIL-10 induction [hIL-10 (reactivated joints)/hIL-10 (unreactivated joints)] in Ad.C3-tat/HIV-hIL-10 injected animals.

ed this in several experiments. In one set of experiments, we compared swelling induced in PG-APS-primed joints by Ad.C3-tat/HIV-luc with that produced by empty Ad.RR5 and Ad.CMV vectors; we found no significant difference (data not shown). In another set of experiments, having compared the severity of PG-APSinduced arthritis recurrence in joints injected with Ad.C3-tat/HIV-luc, Ad.RR5, and saline, we also did not observe significant differences (data not shown). A hightiter (100 PFU/cell) infection of synovial cells in vitro with Ad vectors containing C3-tat/HIV promoter did not cause cytotoxicity (data not shown). As we used low titers in vivo $\left(10^{7} \mathrm{PFU} /\right.$ joint, which in our estimation corresponded to less than $1 \mathrm{PFU} /$ cell in vivo), this makes cytotoxic effects of C3-tat/HIV in vivo unlikely. These observations argue against the cytotoxic and proinflammatory effects of Tat in our experiments. One possible explanation is that many effects of Tat have been attributed to the second exon of tat, which is not present in our construct. Another possibility is that, in the two-component C3-tat/HIV construct, Tat expression is driven by a weak C3 promoter. As we showed previously, even a fully induced C3 promoter provides expression that is two to three orders of magnitude lower than that achieved with strong viral promoters $(11,14)$; therefore the amount of Tat protein should be much less than that described in most experiments using constitutively overexpressed Tat.

In conclusion, our data indicate that the physiologically regulated transgene supplements an inadequate endogenous anti-inflammatory response, thus becoming an integral part of the negative physiological feedback to inflammation. Potential clinical targets for endogenously regulated gene therapy are not limited to 
rheumatoid arthritis; they include a variety of chronic inflammatory diseases, such as Crohn disease and organ transplant rejection (10). It should be possible to regulate the production of many different proteins in this way, using vectors and inflammation-inducible promoters that, like the therapeutic transgene, are tailored to specific clinical settings. The principle of endogenously regulated gene therapy for treating inflammation should therefore not be limited to the promoter system and transgene studied here.

\section{Acknowledgments}

We thank Steven Haskill, Al Baldwin, Michael Brown, and Richard Gaynor for commenting on the manuscript, Roger Brown for preparation of PG-APS, and Julia Romashkova, Dmitry Kovalenko, Steven M. Geiszler, and Abram Trevino for technical assistance. This work was supported by NIH public health grants AR/AI-44564, 5-P60 AR-30701-14, and AR/AI-44030 (to A. Miagkov and S. Makarov); and AI-38596 (to A. Varley and R. Munford). S. Makarov is the recipient of an Investigator Award from The Arthritis Foundation.

1. Evans, C.H., Ghivizzani, S.C., Oligino, T.J., and Robbins, P.D. 2000. Gene therapy for autoimmune disorders. J. Clin. Immunol. 20:334-346.

2. Makarov, S.S. 2000. Gene therapy for rheumatoid arthritis: preclinical studies. In Gene therapy in inflammatory diseases. C.H. Evans and P.D. Robbins, editors. Birkhauser Verlag AG. Basel, Switzerland. 13-34.

3. Muzzonigro, T.S., et al. 2000. Gene therapy for rheumatoid arthritis: clinical studies. In Gene therapy in inflammatory diseases. C.H. Evans and P.D. Robbins, editors. Birkhauser Verlag AG. Basel, Switzerland. 53-64

4. Agha-Mohammadi, S., and Lotze, M.T. 2000. Regulatable systems: applications in gene therapy and replicating viruses. J. Clin. Invest. 105:1177-1183.

5. Clackson, T. 2000. Regulated gene expression systems. Gene Ther. 7:120-125.

6. Ye, X., et al. 1999. Regulated delivery of therapeutic proteins after in vivo somatic cell gene transfer. Science. 283:88-91.

7. Wang, Y., O’Malley, B.W., Jr., Tsai, S.Y., and O'Malley, B.W. 1994. A regulatory system for use in gene transfer. Proc. Natl. Acad. Sci. USA. 91:8180-8184.

8. Rendahl, K.G., et al. 1998. Regulation of gene expression in vivo following transduction by two separate rAAV vectors. Nat. Biotechnol. 16:757-761.

9. Schwab, J.H., Anderle, S.K., Brown, R.R., Dalldorf, F.G., and Thompson, R.C. 1991. Pro- and anti-inflammatory roles of interleukin-1 in recurrence of bacterial cell wall-induced arthritis in rats. Infect. Immun. 59:4436-4442.

10. Varley, A.W., and Munford, R.S. 1998. Physiologically responsive gene therapy. Mol. Med. Today. 4:445-451.

11. Varley, A.W., Geiszler, S.M., Gaynor, R.B., and Munford, R.S. 1997. A twocomponent expression system that responds to inflammatory stimuli in vivo. Nat. Biotechnol. 15:1002-1006.

12. Schwab, J.H. 1995. Bacterial cell wall induced arthritis: models of chronic recurrent polyarthritis and reactivation of monoarticular arthritis. In Mechanisms and models in rheumatoid arthritis. B. Henderson, R. Pettipher, and J. Edwards, editors. Academic Press. London, United Kingdom. 431-446.

13. Makarov, S.S., et al. 1996. Suppression of experimental arthritis by gene transfer of interleukin 1 receptor antagonist cDNA. Proc. Natl. Acad. Sci. USA. 93:402-406.
14. Varley, A.W., Coulthard, M.G., Meidell, R.S., Gerard, R.D., and Munford, R.S. 1995. Inflammation-induced recombinant protein expression in vivo using promoters from acute-phase protein genes. Proc. Natl. Acad. Sci. USA. 92:5346-5350.

15. Makarov, S.S., et al. 1997. NF-kappa B as a target for anti-inflammatory gene therapy: suppression of inflammatory responses in monocytic and stromal cells by stable gene transfer of I kappa B alpha cDNA. Gene Ther. 4:846-852.

16. Miagkov, A.V., et al. 1998. NF-kappaB activation provides the potential link between inflammation and hyperplasia in the arthritic joint. Proc. Natl. Acad. Sci. USA. 95:13859-13864.

17. Lentsch, A.B., Shanley, T.P., Sarma, V., and Ward, P.A. 1997. In vivo suppression of NF- $\kappa B$ and preservation of $I \kappa B \alpha$ by interleukin- 10 and interleukin-13. J. Clin. Invest. 100:2443-2448.

18. Wang, P., Wu, P., Siegel, M.I., Egan, R.W., and Billah, M.M. 1995. Interleukin (IL)-10 inhibits nuclear factor kappa B (NF kappa B) activation in human monocytes. IL-10 and IL-4 suppress cytokine synthesis by different mechanisms. J. Biol. Chem. 270:9558-9563.

19. Apparailly, F., et al. 1998. Adenovirus-mediated transfer of viral IL-10 gene inhibits murine collagen-induced arthritis. J. Immunol. 160:5213-5220.

20. Whalen, J.D., et al. 1999. Adenoviral transfer of the viral IL-10 gene periarticularly to mouse paws suppresses development of collagen-induced arthritis in both injected and uninjected paws. J. Immunol. 162:3625-3632.

21. Fellowes, R., et al. 2000. Amelioration of established collagen induced arthritis by systemic IL-10 gene delivery. Gene Ther. 7:967-977.

22. Müller-Ladner, U., et al. 1999. Gene transfer of cytokine inhibitors into human synovial fibroblasts in the SCID mouse model. Arthritis Rheum. 42:490-497.

23. Lubberts, E., et al. 2000. Intra-articular IL-10 gene transfer regulates the expression of collagen-induced arthritis (CIA) in the knee and ipsilateral paw. Clin. Exp. Immunol. 120:375-383.

24. Schimmer, R.C., et al. 1997. Streptococcal cell wall-induced arthritis. Requirements for neutrophils, P-selectin, intercellular adhesion molecule-1, and macrophage-inflammatory protein-2. J. Immunol. 159:4103-4108.

25. Ghivizzani, S.C., et al. 1998. Direct adenovirus-mediated gene transfer of interleukin 1 and tumor necrosis factor alpha soluble receptors to rabbit knees with experimental arthritis has local and distal anti-arthritic effects. Proc. Natl. Acad. Sci. USA. 95:4613-4618.

26. Otani, K., et al. 1996. Suppression of antigen-induced arthritis in rabbits by ex vivo gene therapy. J. Immunol. 156:3558-3562.

27. Le, C.H., Nicolson, A.G., Morales, A., and Sewell, K.L. 1997. Suppression of collagen-induced arthritis through adenovirus-mediated transfer of a modified tumor necrosis factor alpha receptor gene. Arthritis Rheum. 40:1662-1669.

28. Song, X.Y., Gu, M.L., Jin, W.W., Klinman, D.M., and Wahl, S.M. 1998. Plasmid DNA encoding transforming growth factor- $\beta 1$ suppresses chronic disease in a streptococcal cell wall-induced arthritis model. J. Clin. Invest. 101:2615-2621.

29. Zhang, H., et al. 1997. Amelioration of collagen-induced arthritis by CD95 (Apo-1/Fas)-ligand gene transfer. J. Clin. Invest. 100:1951-1957.

30. Dusetti, N.J., et al. 1997. The pancreatitis-associated protein I promoter allows targeting to the pancreas of a foreign gene, whose expression is up-regulated during pancreatic inflammation. J. Biol. Chem. 272:5800-5804.

31. Kitamura, M., and Kawachi, H. 1998. Creation of an in vivo cytosensor using engineered mesangial cells. Automatic sensing of glomerular inflammation controls transgene activity. J. Clin. Invest. 100:1394-1399.

32. Monahan, P.E., and Samulski, R.J. 2000. Adeno-associated virus vectors for gene therapy: more pros than cons? Mol. Med. Today. 6:433-440.

33. Pan, R.Y., et al. 1999. Disease-inducible transgene expression from a recombinant adeno-associated virus vector in a rat arthritis model. J. Virol. 73:3410-3417.

34. Goater, J., et al. 2000. Empirical advantages of adeno-associated viral vectors in vivo gene therapy for arthritis. J. Rheumatol. 27:983-989.

35. Pan, R.Y., et al. 2000. Therapy and prevention of arthritis by recombinant adeno-associated virus vector with delivery of interleukin-1 receptor antagonist. Arthritis Rheum. 43:289-297. 